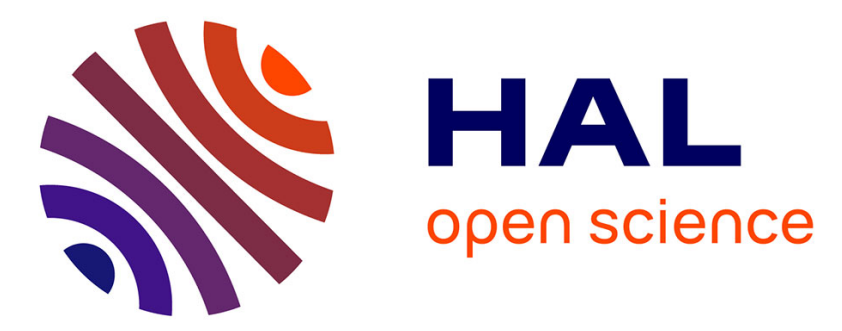

\title{
A narrowband infrared source based on orientation-patterned GaAs for standoff detection of chemicals
}

J. Armougom, M. Melkonian, B. Dherbecourt, M. Raybaut, A. Grisard, E. Lallier, B. Gérard, B. Faure, G. Souhaité, B. Boulanger, et al.

\section{To cite this version:}

J. Armougom, M. Melkonian, B. Dherbecourt, M. Raybaut, A. Grisard, et al.. A narrowband infrared source based on orientation-patterned GaAs for standoff detection of chemicals. Applied Physics B Laser and Optics, 2018, 124 (7), pp.133. 10.1007/s00340-018-7006-x . hal-01842260

\section{HAL Id: hal-01842260 https://hal.science/hal-01842260}

Submitted on 22 Nov 2021

HAL is a multi-disciplinary open access archive for the deposit and dissemination of scientific research documents, whether they are published or not. The documents may come from teaching and research institutions in France or abroad, or from public or private research centers.
L'archive ouverte pluridisciplinaire HAL, est destinée au dépôt et à la diffusion de documents scientifiques de niveau recherche, publiés ou non, émanant des établissements d'enseignement et de recherche français ou étrangers, des laboratoires publics ou privés.

\section{(c) (1) $\$$}

Distributed under a Creative Commons Attribution - NonCommerciall 4.0 International 


\title{
A narrowband infrared source based on orientation-patterned GaAs for standoff detection of chemicals
}

\author{
J. Armougom ${ }^{1} \cdot$ J.-M. Melkonian ${ }^{1} \cdot$ J.-B. Dherbecourt ${ }^{1} \cdot$ M. Raybaut ${ }^{1} \cdot$ A. Grisard $^{2} \cdot$ E. Lallier ${ }^{2} \cdot$ B. Gérard ${ }^{3} \cdot$ B. Faure ${ }^{4}$. \\ G. Souhaité ${ }^{4} \cdot$ B. Boulanger ${ }^{5} \cdot$ A. Godard ${ }^{1}$
}

\begin{abstract}
This paper presents our work on a pulsed single-frequency tunable optical parametric oscillator based on orientation-patterned gallium arsenide. Owing to crystal temperature tuning, this source covers the $10.2-11.2$ and 7.6-8.6 $\mu \mathrm{m}$ ranges in singlelongitudinal mode operation with a linewidth below $30 \mathrm{MHz}$. Standoff detection of ammonia around 10.35 and $10.5 \mu \mathrm{m}$ is performed by implementing this source in an Integrated Path Differential Absorption Lidar (IP-DIAL) setup.
\end{abstract}

\section{Introduction}

Fast and reliable detection of hazardous chemicals or pollutants is an important issue for both civilian and military applications. Among the existing methods, laser infrared spectrometry is promising as it provides non-intrusive and selective standoff detection of chemicals. Active detection systems based on infrared laser spectrometry have already proven their performance for detection of atmospheric pollutants, greenhouse gases, and for industrial purposes [1-6]. In the infrared domain, the longwave infrared (LWIR) region, spanning from 6 to $14 \mu \mathrm{m}$, is of particular interest. Often called the molecular fingerprint region, it gathers the fundamental rovibrational lines of most polyatomic

J.-M. Melkonian

jean-michel.melkonian@onera.fr

1 ONERA-The French Aerospace Lab, 91123 Palaiseau Cedex, France

2 Thales Research and Technology, 1 Avenue Augustin Fresnel, 91767 Palaiseau, France

3 III-V Lab, 1 Avenue Augustin Fresnel, 91767 Palaiseau, France

4 Teem Photonics, 61 Chemin du vieux chêne, 38240 Meylan, France

5 Univ. Grenoble Alpes, CNRS, Grenoble INP, Institut Néel, 38000 Grenoble, France molecules. Within the LWIR region, the third transparency window of the atmosphere ranging from 8 to $12 \mu \mathrm{m}$ allows light to propagate over several kilometers. Standoff detection can then be performed on many species such as $\mathrm{SF}_{6}, \mathrm{HNO}_{3}$, $\mathrm{H}_{2} \mathrm{~S}$, chemical warfare agents (CWAs), toxic industrial chemicals (TICs), explosives and their precursors [7-11].

In the context of standoff detection by infrared spectroscopy, optical coherent sources with narrow spectral linewidth and broad wavelength tunability are attractive to enable selective multi-species detection. At long wavelengths, solid-state materials suitable as laser active ion hosts are scarce, as they usually present strong multiphonon absorption. Only two types of laser technologies are available for direct emission in the LWIR: the $\mathrm{CO}_{2}$ gas laser and the quantum cascade laser (QCL). Until the advent of QCLs, the $\mathrm{CO}_{2}$ laser has been the only coherent source available in the LWIR with a significant energy, and has been extensively used in lidar emitters for standoff detection of chemicals. Several emitters delivering more than $100 \mathrm{~mJ}$ have been developed, mainly for detection of CWAs and TICs [7, 12-14]. Energies above $1 \mathrm{~J}$ have been demonstrated in operational lidars $[15,16]$ while the smallest systems have led to military-grade products [17]. More recently, the $\mathrm{CO}_{2}$ laser has also been used for standoff detection of explosives [18, 19]. However, its restricted tunability still prevents addressing the strongest absorbing bands of most gases, and sometimes precludes detection of the species at all, sulfur mustard for instance. QCLs are now the workhorses of many spectroscopy and local gas sensing experiments [20]. However, due to their limited peak power, standoff detection with QCL is currently limited to ranges of 
a few tens of meters using reflectors or topographical targets. Nevertheless, advanced developments have been carried out by several groups on standoff detection of explosives on contaminated surfaces [21-23] and on standoff detection of liquid or gaseous hazardous chemicals [24-27]. A review on photoacoustic detection of explosives using QCLs can be found in Ref. [28]. To overcome these limitations, another solution is to use second-order nonlinear optics to achieve down-conversion to the LWIR. For instance, optical parametric oscillators (OPOs) are well suited for standoff detection of chemicals because they provide peak powers in the kilowatt range, greater wavelength tunability compared to QCLs and $\mathrm{CO}_{2}$ lasers and good optical quantum efficiencies (typically around 30\% with Gaussian beams). Most of previous research studies focused on pumping crystals such as $\mathrm{AgGaSe}_{2}, \mathrm{BaGa}_{4} \mathrm{~S}_{7}, \mathrm{ZnGeP}_{2}, \mathrm{CdSe}$ and orientation-patterned GaAs (OP-GaAs) where wavelength tunability can reach a few micrometers in the LWIR [29-37]. Among these crystals, GaAs is one of the most interesting for parametric frequency conversion thanks to the following features: a broad transparency window ranging from 0.9 to $17 \mu \mathrm{m}$, a high second order nonlinear susceptibility $\left(d_{14} \sim 90 \mathrm{pm} \mathrm{V}^{-1}\right)$ [38], and excellent mechanical and thermal properties. GaAs is optically isotropic, precluding birefringent phase-matching, but it is free from spatial walk-off which usually limits the maximum usable length. Efficient conversion in GaAs is obtained with quasi phase-matched (QPM) crystals where the sign of the second-order nonlinear susceptibility is periodically inverted to compensate for phase velocity mismatch between the nonlinear polarization and the radiated field. Initially, QPM GaAs crystals were obtained by stacking several thin plates of GaAs that were bonded by diffusion of adjacent layers, but these structures suffered from scattering losses at domain interfaces [39]. More recently, orientation-patterned GaAs based on epitaxial growth solved these problems and offered more efficient crystals for parametric conversion [40-45].

In this paper, we present our most recent work on the first pulsed single longitudinal mode and wavelength tunable LWIR optical parametric oscillator based on OP-GaAs. Initial results regarding this source have already been published in Ref. [46] but significant efforts were still needed to fully characterize it and to efficiently adapt its design for relevant applicative demonstrations. Here we carry out an in depth analysis of the wavelength tunability, gain bandwidth and linewidth measurements. We also give more insights on the source design and a discussion on its implementation in an integrated-path lidar (IP-DIAL). In Sect. 2, we present the unique OPO architecture developed by our team-called NesCOPO - that ensures single longitudinal mode emission as well as wide wavelength tunability. Then in Sect. 3, we present the source performance in terms of energy, oscillation threshold, tunability and linewidth. Finally in Sect. 4, we implement this source in an IP-DIAL setup and perform standoff detection of ammonia around $10.5 \mu \mathrm{m}$.

\section{Nested cavity OPOs: working principle}

Nested cavity OPOs (NesCOPOs) are doubly-resonant OPOs with physically separated cavities for the idler and signal waves [47]. This architecture combines low oscillation threshold thanks to its doubly-resonant and doublepass architecture, a broad frequency tunability within the gain bandwidth with fine adjustment of cavity lengths, as well as single-mode operation without any injection seeding or intracavity filters. Figure 1 shows a scheme of the NesCOPO used in our setup. As depicted, idler and signal waves oscillate respectively between mirrors $\mathrm{M}_{1}-\mathrm{M}_{3}$ and $\mathrm{M}_{2}$ $-\mathrm{M}_{3}$. Here, $\mathrm{M}_{3}$ is common to both cavities and reflects the pump back into the crystal. This leads to a lower threshold and reduces parasitic Fabry-Perot effects [48]. Under the condition that the nonlinear phase is maintained through the double-pass, a better mode selectivity due to parametric gain bandwidth narrowing as well as better conversion efficiency are achieved [49]. This architecture has been mainly implemented with ferroelectric crystals such as periodically poled lithium niobate (PPLN), for gas sensing applications between 1.5 and $4.2 \mu \mathrm{m}[50,51]$. Here, this concept is extended to the LWIR using orientation-patterned gallium arsenide (OP-GaAs) crystals.

At first order and far from degeneracy the theoretical double-pass gain bandwidth is given by:

$\Delta \sigma=\frac{1}{2 L \cdot\left(n_{\mathrm{g}, \mathrm{s}}-n_{\mathrm{g}, \mathrm{i}}\right)}$,

where $L$ is the crystal length, $n_{\mathrm{g}}$ stands for the group index, and the subscripts $\mathrm{s}$, i for the signal and idler waves respectively. In our case, the theoretical gain bandwidth is $8.5 \mathrm{~cm}^{-1}$ for a $10 \mathrm{~mm}$ long OP-GaAs crystal using the group indices given in Ref. [38]. Without any spectral selection mechanism, as in a singly resonant OPO, there would be approximately 80 longitudinal modes that could oscillate under the gain bandwidth with a cavity free spectral range (FSR) of

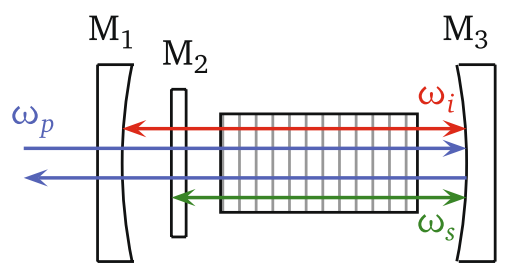

Fig. 1 Scheme of a NesCOPO in double-pass configuration. Pump in blue, idler in red and signal in green. The idler cavity is formed by $M_{1}$ $-\mathrm{M}_{3}$ and the signal cavity by $\mathrm{M}_{2}-\mathrm{M}_{3}$ 
(a)

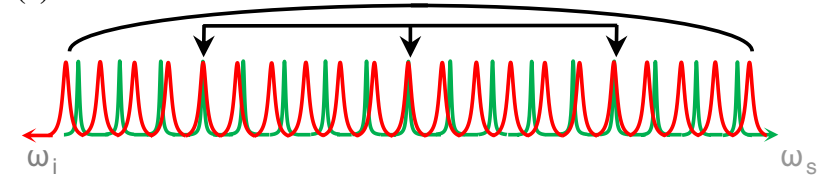

(b)
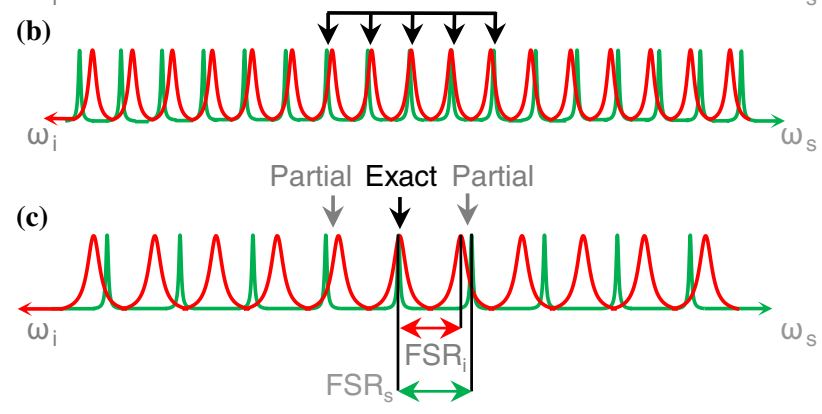

Fig. 2 Illustration of mode selection in a NesCOPO using Giordmaine and Miller's diagram [52]. Idler (red) and signal (green) axes are in opposite direction so that a vertical line fulfills energy conservation: $\omega_{\mathrm{p}}=\omega_{\mathrm{s}}+\omega_{\mathrm{i}}$. FSR stands for free spectral range. a High dissociation: several exact coincidences under gain bandwidth (multimode emission). b Low dissociation: emission of clusters (multimode emission). c Intermediate dissociation: one exact coincidence under the gain bandwidth (single-mode emission)

$4 \mathrm{GHz}$. However, in a NesCOPO, the signal and the idler waves oscillate in their respective cavities resulting in two resonant mode combs, as illustrated in Fig. 2. As shown in this figure, idler (red) and signal (green) axes are in opposite direction so that a vertical line fulfills energy conservation: $\omega_{\mathrm{p}}=\omega_{\mathrm{s}}+\omega_{\mathrm{i}}$. There is an exact coincidence when an idler mode matches a signal mode with maximum overlap. Mathematically, the exact double oscillation condition can be written as follows :

$\left\{\begin{array}{l}\omega_{\mathrm{s}}=q_{\mathrm{s}} \cdot \frac{\pi c}{L_{\mathrm{s}}} \\ \omega_{\mathrm{i}}=q_{\mathrm{i}} \cdot \frac{\pi c}{L_{\mathrm{i}}}\end{array}\right.$,

where $L_{\mathrm{s}, \mathrm{i}}$ are the optical lengths of the two cavities, $q_{\mathrm{s}, \mathrm{i}}$ being integers and $\omega_{\mathrm{p}}=\omega_{\mathrm{s}}+\omega_{\mathrm{i}}$. However, due to the limited cavity finesse, partial coincidences can appear within the gain bandwidth when the overlap between neighboring modes is sufficient to initiate oscillation, leading to multimode operation.

In a NesCOPO, spectral content of the emission can be controlled by adjusting the dissociation parameter which is defined as follows [47]:

$\frac{\Delta L}{L}=\frac{L_{\mathrm{s}}-L_{\mathrm{i}}}{L_{\mathrm{s}}}$,

where $L_{\mathrm{s}, \mathrm{i}}$ are the group cavity lengths at the signal and idler waves respectively, and $L$ can be taken as $L_{\mathrm{s}}$ as a convention.
Assuming a negligible dispersion, the spectral gap between two exact coincidences is:

$\Delta \omega_{\mathrm{s}, \mathrm{i}} \simeq \frac{L}{\Delta L} \cdot \frac{\pi c}{n_{\mathrm{g},\{\mathrm{s}, \mathrm{i}\}} \mathrm{L}}=\frac{L}{\Delta L} \cdot 2 \pi \mathrm{FSR}_{\{\mathrm{s}, \mathrm{i}\}}$,

with $n_{\mathrm{g},\{s, i\}}$ standing for the group index of the waves and $\mathrm{FSR}_{\{s, i\}}$ are the free spectral range of the cavities. According to Eq. (4), coincidences are closer to each other when the dissociation parameter is high, leading to several exact coincidences under the gain bandwidth (Fig. 2a). Conversely, the NesCOPO emits a cluster of modes including multiple partial coincidences for a low dissociation (Fig. 2b). In both cases, the emitted spectrum is multimode. To operate in single-longitudinal-mode, it is thus essential to choose an intermediate value for $\Delta L / L$ (Fig. 2c). To calculate the adequate dissociation parameter for a $10 \mathrm{~mm}$ long OP-GaAs crystal, we followed the procedure given in Ref. [53]. We considered calculated finesse coefficients of 28 and 11 for the signal and idler cavity, respectively, using the measured transmission curves provided by the coating company. We used an experimental value of the gain bandwidth of $10.5 \mathrm{~cm}^{-1}$ and a moderate pumping intensity two times above threshold for an exact coincidence. In Fig. 3, we plotted the maximum overlap integral between the signal and idler Fabry-Perot longitudinal cavity modes as a function of the dissociation parameter $\Delta L / L$. There, the red line represents the threshold above which partially overlapping neighbouring modes can oscillate for the chosen pumping rate. From Fig. 3, it can be seen that the multi-frequency behaviour shown in Fig. 2a) is obtained in region (3) for a high value of the dissociation parameter. As well, a cluster of modes (Fig. 2b) is obtained

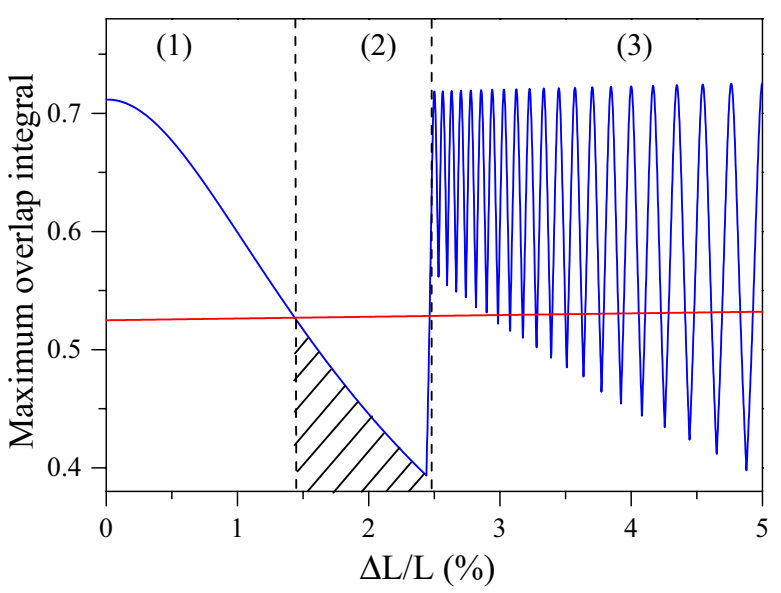

Fig. 3 Maximum overlap integral between idler and signal modes as a function of the dissociation parameter $\Delta L / L$ for a $10 \mathrm{~mm}$ long OPGaAs crystal. The red line corresponds to the overlap above which neighbouring modes can oscillate for a pumping rate of 2. Region (1): a cluster of modes is emitted, region (2): single-frequency operation, and region (3): multimode operation (with several exact coincidences) 
when the dissociation parameter is in region (1). On the contrary, when the dissociation parameter is set around a few percent [region (2)], it is possible to achieve singlefrequency operation. Note that for a $10 \mathrm{~mm}$ long crystal, the OP-GaAs already introduces a chromatic dissociation of $+1.6 \%$, close to the optimum. Thus the signal and idler cavity physical lengths need to be very close to each other. To make this possible, the signal mirror has been deposited on the outer face of mirror $\mathrm{M}_{2}$ [46]. We will explain in Sect. 3.4 how the optimum dissociation value is obtained experimentally.

Once the physical difference between the signal and idler cavities has been set, fine wavelength tuning can be done by adjusting one or both cavity lengths using piezo-electric transducers. As can be seen from Eq. (2), changing one cavity length is equivalent to stretching the associated frequency comb. At first order and at the scale of a few modes, this effect can be approximated to a translation in the Giordmaine and Miller's diagram. In a NesCOPO, fine adjustment of the cavity lengths are obtained with mirrors mounted on piezo-electric transducers. A displacement of one of the mirrors only changes one of the two cavity lengths, inducing mode hops within the gain bandwidth, so that a discrete set of wavelengths is emitted sequentially. When the second mirror is translated, the other cavity length is changed, creating a new set of accessible wavelengths. This mechanism is the optical analog of the mechanical Vernier. For broader tunability, the nonlinear crystal can be heated to shift the gain bandwidth.

\section{Implementation of the LWIR source}

\subsection{OP-GaAs crystals characterization}

We used three OP-GaAs samples (namely M19, M28 and M22) whose characteristics are presented in Table 1. They are periodically oriented and grown by epitaxy in vapor phase [40]. In this method, two layers of GaAs with opposite crystallographic directions [001] and [00 - 1] are superimposed

Table 1 Specifications of the OP-GaAs crystals

\begin{tabular}{|c|c|c|c|c|c|}
\hline \multirow[t]{2}{*}{ Crystal } & \multirow[t]{2}{*}{ M19 } & \multirow[t]{2}{*}{ M28 } & \multicolumn{3}{|c|}{ M22 (multi-tracks) } \\
\hline & & & $\Lambda_{1}$ & $\Lambda_{2}$ & $\Lambda_{3}$ \\
\hline Length (mm) & 10 & 6 & 10 & & \\
\hline QPM period $(\mu \mathrm{m})$ & 72.6 & & 66 & 64.8 & 63.6 \\
\hline Idler $(\mu \mathrm{m})$ & $10.2-11.2$ & & $8.3-9$ & $7.9-8.7$ & $7.6-8.3$ \\
\hline $\begin{array}{l}\text { Focusing parameter } \\
\text { (idler) }\end{array}$ & 0.73 & 0.54 & 0.59 & 0.57 & 0.54 \\
\hline $\begin{array}{l}\text { Focusing parameter } \\
\text { (signal) }\end{array}$ & 0.34 & 0.25 & 0.36 & 0.37 & 0.37 \\
\hline
\end{tabular}

on a GaAs wafer. The domain periods and duty cycles are defined by photolithography, and the patterned template is etched to reveal the grating. Finally, the material grows on about $500 \mu \mathrm{m}$ by epitaxy in vapor phase. The crystal's facets are then anti-reflection coated for the pump, signal and idler waves. Pictures of these crystals are shown in Fig. 4 where the crystallographic orientation is shown in the first inset. The surface damage threshold was measured to be approximately $0.6 \mathrm{~J} \mathrm{~cm}^{-2}$ at $2 \mu \mathrm{m}$ for anti-reflection coated crystals.

In a preliminary experiment, we evaluated the temperature acceptance of crystal M28 at $10.7 \mu \mathrm{m}$ by difference frequency generation between the signal and the idler waves generated by a type II PPLN OPO. This OPO is pumped at $1.064 \mu \mathrm{m}$ by a Nd:YAG multimode laser. Thanks to type II phase-matching the idler and signal linewidths are both close to $1 \mathrm{~cm}^{-1}$. The emitted wavelengths of the OPO were set to $1.936 \mu \mathrm{m}$ for the signal and $2.362 \mu \mathrm{m}$ for the idler. Figure 5 shows the difference-frequency generation efficiency of the OP-GaAs crystal as a function of its temperature (blue) and the theoretical model (red). In the undepleted pump approximation, the difference frequency generation intensity is proportional to:

$I_{\mathrm{DFG}}(T) \propto \operatorname{sinc}^{2}\left(\frac{\Delta k(T) \cdot L}{2}\right)$,

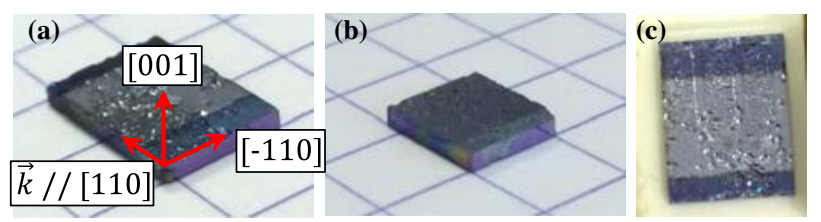

Fig. 4 Pictures of the OP-GaAs crystals used in the experiment. a M19 with the crystallographic orientation, b M28 and $\mathbf{c}$ M22

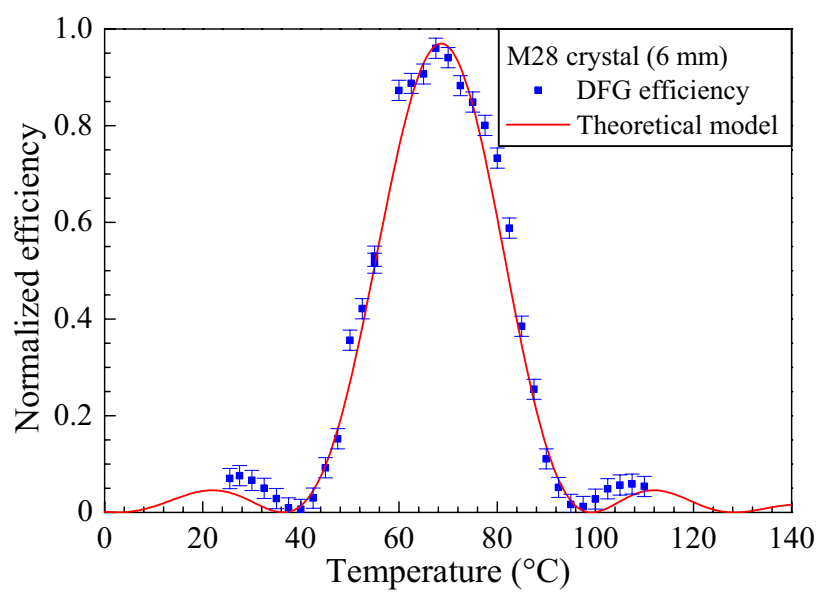

Fig. 5 Difference-frequency generation efficiency versus OP-GaAs temperature at $\lambda_{\mathrm{s}}^{\mathrm{PPLN}}=1.936 \mu \mathrm{m}$ and $\lambda_{\mathrm{i}}^{\mathrm{PPLN}}=2.362 \mu \mathrm{m}$ for M28. In blue, measurement data and in red theoretical model 
where $\Delta k(T)$ is the temperature dependant quasi-phase mismatch and $L$ is the length of the OP-GaAs crystal. Calculation on the theoretical model is carried out using the refractive index equations provided in Ref. [38]. As we can see in Fig. 5, the width of the efficiency curve is in very good agreement with the theoretical expectation. Moreover, the existence of true zeros (at 40 and $100^{\circ} \mathrm{C}$ ) suggest that the grating has few periodicity defects [54].

\subsection{NesCOPO experimental setup}

The experimental setup is shown in Fig. 6. The OPO is pumped by a single-frequency pulsed $\mathrm{Tm}^{3+}$ :YAP microlaser emitting at $1938.5 \mathrm{~nm}$ specifically designed and ruggedized for this application [55]. It delivers pulses of $36 \mathrm{~ns}$ with a repetition rate of $100 \mathrm{~Hz}$. A maximum output energy of $170 \mu \mathrm{J}$, corresponding to $4.7 \mathrm{~kW}$ peak power per pulse was achieved. The laser rod is a $4-\mathrm{mm}$ long $3 \%$ at. Tm:YAP, cut along the crystallographic a-axis. The cavity input mirror has been deposited on one facet of the rod (HT@795 nm, HR@1940 nm) to reduce the number of elements in the cavity. The laser crystal is pumped by a $3 \mathrm{~W}$ fibered laser diode at $793 \mathrm{~nm}$. Passively Q-switch operation is obtained with a $\mathrm{Cr}^{2+}: \mathrm{ZnSe}$ saturable absorber. To avoid mode hopping, a $125-\mu \mathrm{m}$-thick YAG etalon was inserted into the cavity, and single-longitudinal mode operation was eased with a pump modulation scheme. The output beam is nearly diffraction limited with a $\mathrm{M}^{2}$ parameter of 1.02. An optical isolator is placed on the beam path to prevent from optical feedback. Two half-wave plates are inserted before and after the isolator to, respectively, adjust the pump power and polarization. Then, the pump beam is focused with a $150-\mathrm{mm}$ focal length $\mathrm{CaF}_{2}$ lens into the OP-GaAs crystal. The beam waist radius at $1 / \mathrm{e}^{2}$ was measured to be $100 \mu \mathrm{m}$. A ZnSe mirror reflecting $98 \%$ of the signal wave, a prismatic $\mathrm{CaF}_{2}$ plate, and a dichroic plate in $\mathrm{ZnSe}$ are inserted between the lens and the OPO to separate the signal, the reflected pump and the idler waves. The idler cavity consists of a spherical mirror $\mathbf{M}_{1}$ of

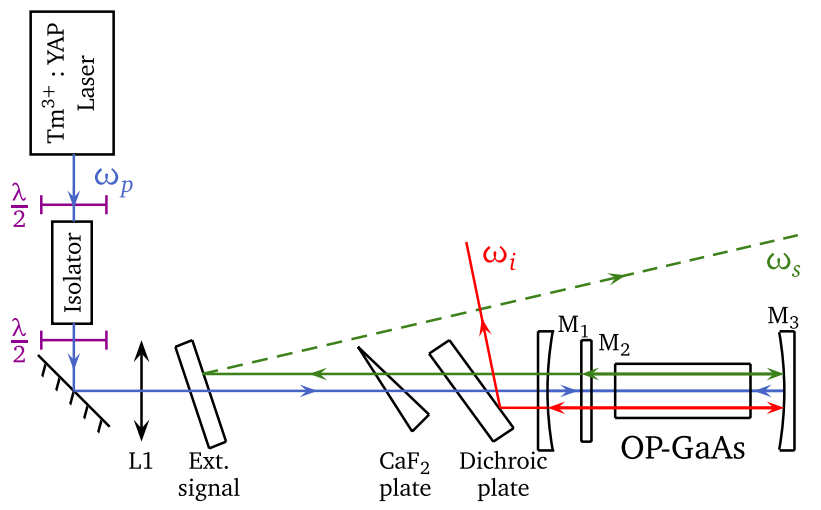

Fig. 6 Experimental setup of the NesCOPO emitter
$20 \mathrm{~mm}$ radius of curvature, reflecting $85 \%$ of the idler in the 8-11 $\mu \mathrm{m}$ range and a gold-coated mirror $\mathrm{M}_{3}$ of $50 \mathrm{~mm}$ radius of curvature. The signal cavity consists of the same mirror $\mathrm{M}_{3}$ and a plane mirror $\mathrm{M}_{2}$ reflecting $98 \%$ at the signal wavelength. For M19 and M22, we calculated beam waists radii at $1 / \mathrm{e}^{2}\left(w_{0}\right)$ of $105 \mu \mathrm{m}$ for the signal mode and $150 \mu \mathrm{m}$ for the idler mode. For M28, the calculated beam waists radii are $95 \mu \mathrm{m}$ for the signal and $135 \mu \mathrm{m}$ for the idler. The focusing parameters of each cavity, which writes $\xi=L /\left(2 \pi \frac{w_{0}^{2}}{\lambda}\right)$, are shown in Table 1 . The crystal is inserted between $\mathbf{M}_{2}$ and $\mathrm{M}_{3}$ in a temperature-controlled oven. Experimentally, we could test each crystal within the same cavity. $\mathrm{M}_{1}$ and $\mathrm{M}_{3}$ are mounted on piezo-electric transducers to finely adjust cavity lengths. The free spectral range is approximately $4 \mathrm{GHz}$ for each cavity.

\subsection{Source efficiency}

We obtained typical oscillation thresholds of $2 \mu \mathrm{J}$ for M22, $5.5 \mu \mathrm{J}$ for M19 and $6.2 \mu \mathrm{J}$ for M28 after optimization of the NesCOPO alignment and lateral position of the crystal. We also set the pump polarization along [-110] in order to minimize the oscillation threshold which gives a measured idler polarization along [-110]. The threshold values are still two to three times higher than theoretical expectations. We attribute this discrepancy to losses due to small beam clipping of the LWIR beam by the crystal edges and imperfect dielectric coatings. Figure 7 shows the measured idler energy as a function of the incident pump energy for each NesCOPO configuration and Table 2 summarizes their efficiency.

Three times above threshold, we achieved a typical energy of $150 \mathrm{~nJ}$ output power at the idler wavelength with a maximum of $250 \mathrm{~nJ}$ for M19 which correspond to a peak power of $5.5 \mathrm{~W}$. The pulse-to-pulse energy fluctuation of the idler is $\pm 1 \%$ rms over $1 \mathrm{~s}$. As seen from Fig. 7a), M19 has a lower slope efficiency than M28. This discrepancy can be explained by greater beam clipping at the crystals ends in longer crystals. Moreover, M19 present small imperfections as only a few localized spots in the crystal leads to OPO oscillation. In addition, these spots were damaged by the laser during preliminary experiments. In M28, oscillation can be obtained anywhere in the crystal. In Fig. 7b), at high pumping rates we can see a gap between the three curves. We attribute this gap to multimode operation of the NesCOPO which typically occurs over three times above threshold $(\sim 6$ $\mu \mathrm{J}$ here). Figure 8 shows the temporal pulse profiles of the incident and depleted pump as well as the idler profile for M19. We determined an average pump depletion of $15 \%$ with a pump energy four times above threshold. Pumping more than three to four times above threshold is not advised as it can lead to multimode operation due to less efficient Vernier spectral filtering of the side-modes. 

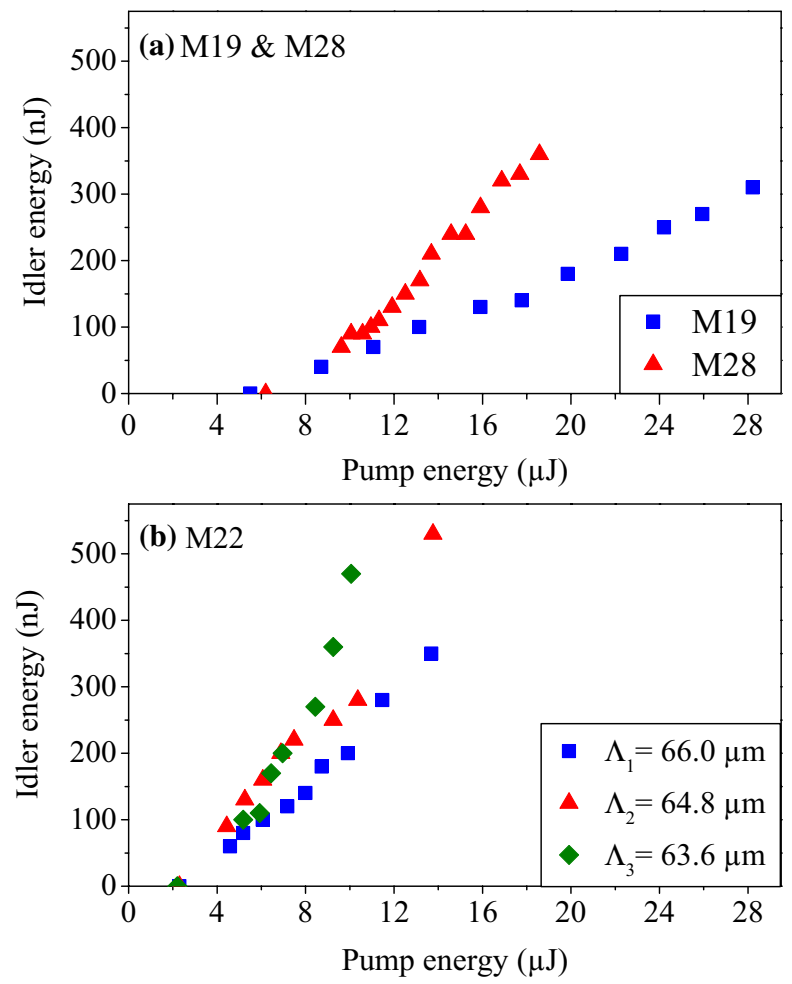

Fig. 7 Idler energy as a function of the incident pump energy for each crystal. a M19 for an idler wavelength set around $10.4 \mu \mathrm{m}$ (blue) and M28 for an idler wavelength set around $10.2 \mu \mathrm{m}$ (red). b M22, $\Lambda_{1}=66 \mu \mathrm{m}$ for an idler wavelength set around $8.3 \mu \mathrm{m}$ (blue), $\Lambda_{2}=64.8 \mu \mathrm{m}$ for an idler wavelength set around $7.9 \mu \mathrm{m}$ (red) and $\Lambda_{3}=63.6 \mu \mathrm{m}$ for an idler wavelength set around $7.6 \mu \mathrm{m}$ (green)

Table 2 NesCOPO efficiency and pump depletion for each crystal

\begin{tabular}{llllll}
\hline Crystal & M19 & M28 & M22 & & \\
\hline QPM period $(\mu \mathrm{m})$ & 72.6 & 72.6 & 66 & 64.8 & 63.6 \\
Threshold $(\mu \mathrm{J})$ & 5.5 & 6.2 & 2.3 & 2.3 & 2.2 \\
LWIR energy $(\mathrm{nJ})$ & 250 & 120 & 100 & 150 & 150 \\
Pump depletion $(\%)$ & 18 & 10 & 16 & 16 & 23 \\
\hline
\end{tabular}

The output energy is measured for a pump ratio of three, after the dichroïc plate and a long-pass filter at $6840 \mathrm{~nm}$ to remove residual pump and signal waves while the pump depletion is measured for a pump ratio of four

\subsection{Single frequency operation and wavelength tunability}

As explained in Sect. 2, single-frequency operation is achieved when the dissociation parameter $\Delta L / L$ is adjusted to avoid both multiple exact coincidences and clusters of modes under the gain bandwidth. Experimentally, the idler's cavity length is mechanically adjusted while spectrum analysis is performed with a scanning Fabry-Perot interferometer to verify that the OPO output is single-frequency. For this purpose the signal beam is filtered with a highly reflective

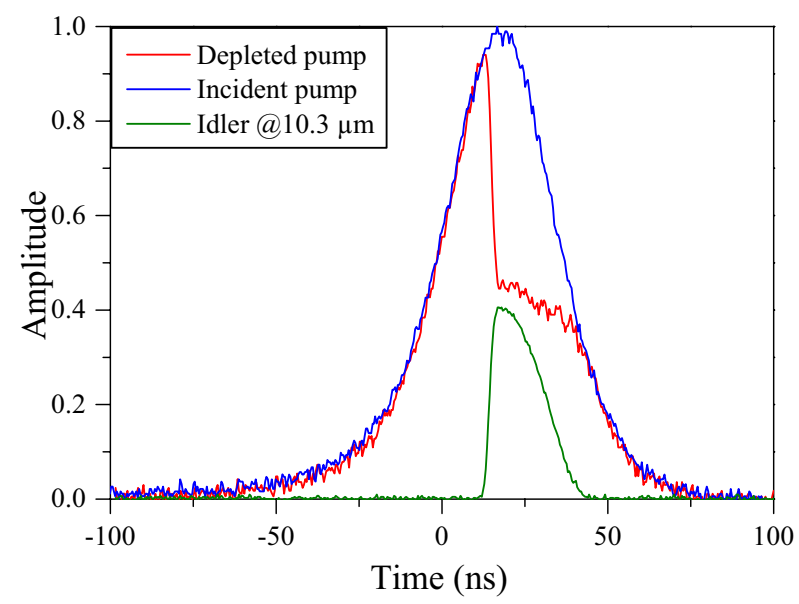

Fig. 8 Temporal pulse profiles of the pump at the entrance (blue) and at the exit (red) of the NesCOPO, and of the idler for M19 measured four times above threshold

mirror at the signal wavelength and a long-pass filter above $2000 \mathrm{~nm}$ and fiber-coupled into the interferometer. The output signal is detected with an InGaAs photodiode. The Fabry-Perot free spectral range (FSR) is $10 \mathrm{GHz}$, which is well adapted to detect side-modes from the NesCOPO output since the signal and idler cavities FSR are close to 4 GHz. In Fig. 9 (top), under the setpoint ramp (blue), we can see two peaks separated by the interferometer's FSR (red) showing that the NesCOPO is single-longitudinal mode. For a multimode NesCOPO, the graph would show several peaks separated by a multiple of the NesCOPO FSR such as in Fig. 9 (bottom). We can see a new peak separated from the last one by $4 \mathrm{GHz}$ that is the NesCOPO FSR. To ensure that single-mode operation was always obtained for the chosen fixed dissociation value, we repeated this measurement for every accessible wavelength under the gain bandwidth, and for each OP-GaAs crystal. After this adjustment procedure, we physically measured the cavity lengths and estimated the dissociation parameter to be between 1 and $2 \%$ for a $10 \mathrm{~mm}$ long crystal which is consistent with Fig. 3.

Besides single-frequency operation, it is necessary to estimate the gain bandwidth and the tunability of the NesCOPO. The width of the gain bandwidth is indeed the ultimate limit for fast (e.g., pulse to pulse) wavelength tunability at set pump wavelength and crystal temperature: it is thus interesting for designing a differential absorption lidar system. We injected the signal wave in an optical spectrum analyzer (Yokogawa) to estimate the gain bandwidth: the resolution was $3 \mathrm{GHz}(0.05 \mathrm{~nm})$ and the apparatus could span from 1200 to $2400 \mathrm{~nm}$. For this measurement, both cavity mirrors are rapidly swept so that all the longitudinal modes within the gain bandwidth are sequentially emitted during the integration time of the spectrum analyzer. The first inset of Fig. 10 shows these results for M19 at a 

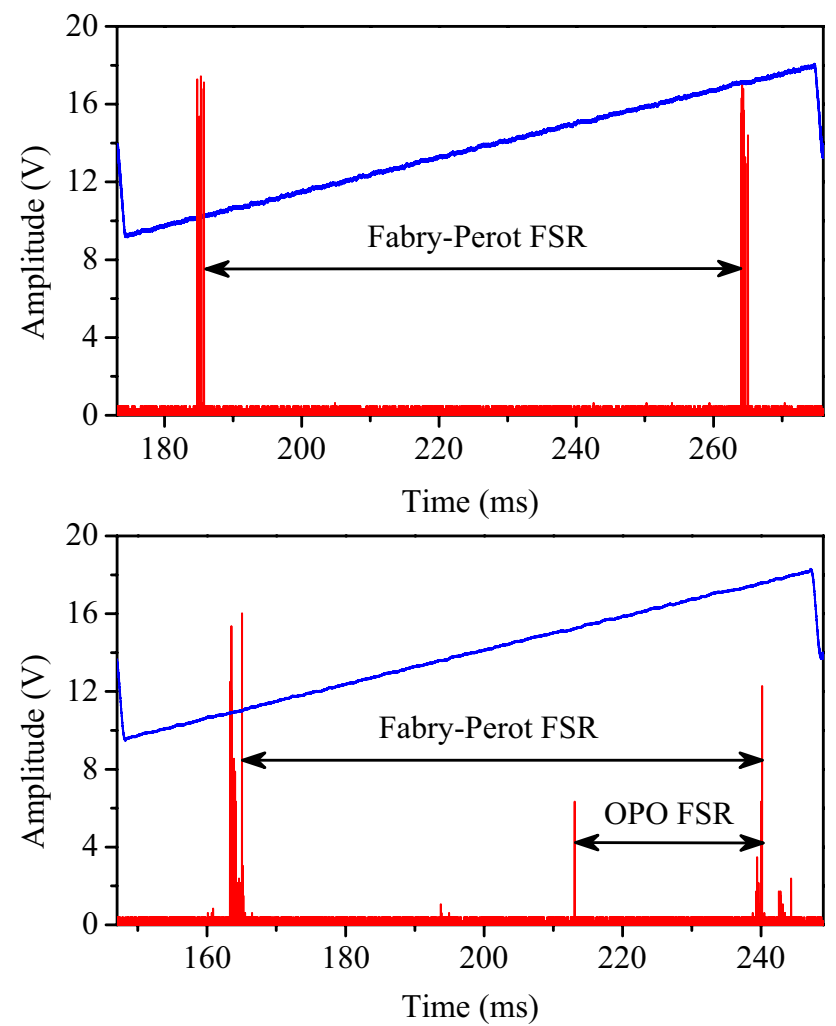

Fig. 9 Signal spectrum analyzed with the scanning Fabry-Perot interferometer for single-frequency emission (top) and multi-frequency emission (bottom). In blue, the setpoint ramp and in red, the spectrum

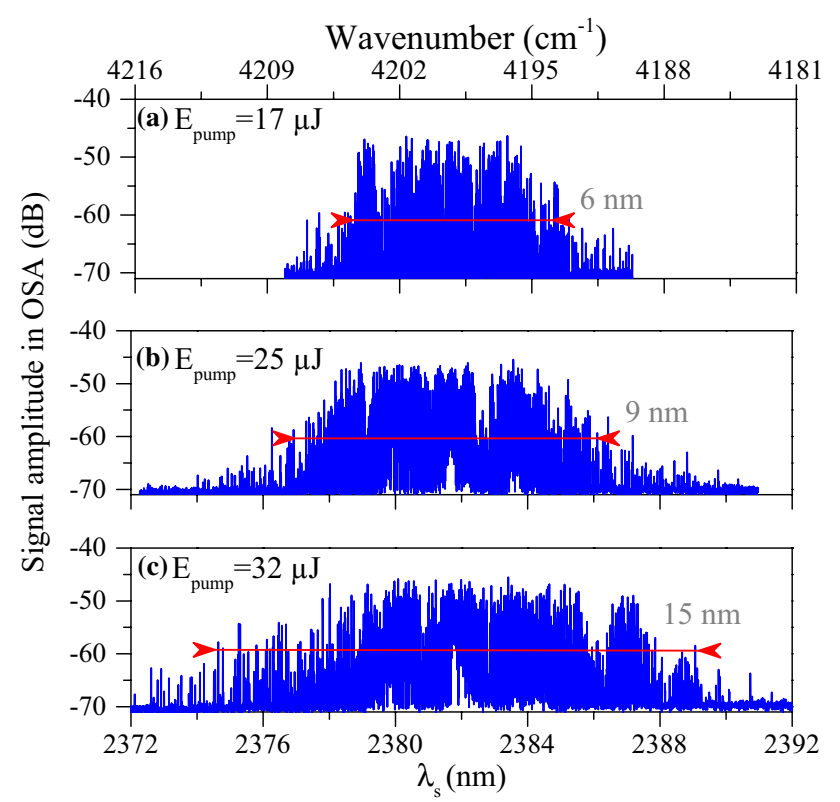

Fig. 10 Shape of the gain bandwidth centered at $\lambda_{\mathrm{s}}=2381.5 \mathrm{~nm}$ for M19 emitter. a $E_{\text {pump }}=17 \mu \mathrm{J}, \mathbf{b} E_{\text {pump }}=25 \mu \mathrm{J}$ and $\mathbf{c} E_{\text {pump }}=32 \mu \mathrm{J}$. These energies correspond to pumping rates of $3.5,5$ and 6.5 , respectively central wavelength of $2381.5 \mathrm{~nm}$, and for a pumping rate of 3.5 above threshold. The measured spectral envelope gives the wavelength bandwidth accessible with the NesCOPO at a given temperature and pump wavelength. The spectral bandwidth has a width of $6 \mathrm{~nm}$ at the signal wavelength, which corresponds to $10.5 \mathrm{~cm}^{-1}$. This value is consistent with the theoretical double-pass first-order gain bandwidth of $8.5 \mathrm{~cm}^{-1}$ (Eq. 1). Moreover the spectral bandwidth is monolobe, suggesting that the relative phase of the waves, i.e., $\Delta \varphi=\varphi_{\mathrm{p}}-\varphi_{\mathrm{s}}-\varphi_{\mathrm{i}}$, in the backward trip after reflection by $M_{3}$ is equal to the ideal value of $-\pi / 2$ [49]. Since the gold coating of $\mathrm{M}_{3}$ introduces a $\pm \pi$ relative dephasing between the three waves, and assuming that antireflective coatings have a negligible contribution, this means that the output end of the nonlinear crystal ends nearly at half a quasi-phasematching period. To control perfectly the backward relative phase, future improvements will include a wedge on the output facet of the nonlinear crystal, as we already did in PPLN-based NesCOPOs [56]. Pumping six and half times above threshold broadens the spectral bandwidth to $15 \mathrm{~nm}$. But then the output becomes multimode because the gain for the side-modes becomes too high to ensure successful Vernier spectral filtering. This is equivalent to lowering the red line in Fig. 3. In the following, the NesCOPO is pumped three times above threshold.

The idler wavelength of the OPO is tunable over $35 \mathrm{~nm}$ thanks to piezo-electric transducers. However, to perform broader tunability, it is necessary to change the crystal temperature to shift the gain bandwidth toward higher or smaller central wavelengths. Figure 11 shows this shift in wavelength for each crystal. For M19 and M28, we achieved a tunability from 10.2 to $11.2 \mu \mathrm{m}$ by heating the crystal up to $90^{\circ} \mathrm{C}$ which corresponds to a rate of $9.9 \mathrm{~nm}^{\circ} \mathrm{C}^{-1}$. This tuning range allows many chemical species to be detected such as $\mathrm{NH}_{3}, \mathrm{SF}_{6}$, sarin or triethyl phosphate (TEP) which

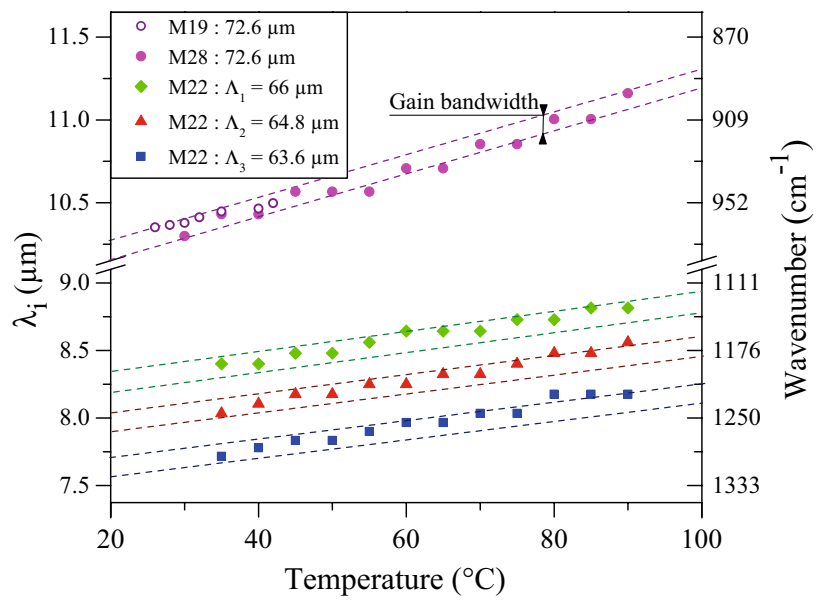

Fig. 11 Idler wavelength as a function of crystal temperature. Points are measured data and dashed lines represent the gain bandwidth 
is a simulant for the stretching vibration of sarin's $\mathrm{P}-\mathrm{O}-\mathrm{C}$ bond [57]. Using M22, we reached $8 \mathrm{~nm}^{\circ} \mathrm{C}^{-1}$ for each track leading to a tunability of $1 \mu \mathrm{m}$ from 7.56 to $8.56 \mu \mathrm{m}$, a region which includes the main absorption band of sulfur mustard [57].

\subsection{Wavelength and linewidth measurements by frequency beating with a continuous-wave source}

To carry out an investigation of the spectral properties of the NesCOPO pulses, we realized optical heterodyne measurements and analysed the beat-note signal between the OPO and a stabilized continuous-wave QCL. Here, heterodyne detection has the advantage to give the information on the instantaneous spectral properties of the source whereas other measurement apparatus such as a Fizeau lambda meter or a monochromator would, respectively, give the mean wavelength or the spectrum averaged over several hundreds of pulses. Figure 12 shows the experimental setup used for optical heterodyne measurement. The local oscillator is a commercial CW distributed feedback (DFB) QCL tunable around $10.33 \mu \mathrm{m}$ (AdTech optics). The QCL beam is divided in two parts with a beamsplitter (BS1). The first part goes onto a commercial lambdameter (High Finesse) for wavelength measurement while the second part is mixed with the idler beam. The idler pulse and the QCL are combined on a $\mathrm{HgCdZnTe}$ photoelectromagnetic detector (Vigo) whose bandwidth is close to $500 \mathrm{MHz}$. The idler beam is also divided in two parts with another beamsplitter (BS2), the first part is mixed with the QCL beam and the second part goes into a monochromator (Jobin Yvon) and is detected by a nitrogen cooled $\mathrm{HgCdTe}$ detector for wavelength measurement.

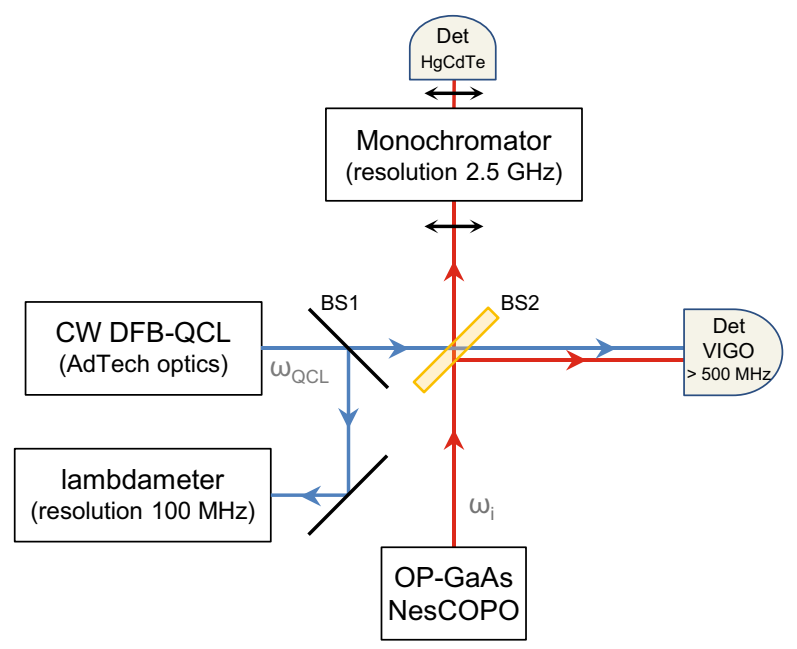

Fig. 12 Heterodyne detection scheme
For the experiment, we tuned the QCL wavelength at $10337.56 \mathrm{~nm}$ which is one of the most intense lines of the ammonia absorption band around $10.3 \mu \mathrm{m}$ which we are going to detect in Sect. 4.3. Then, we set the temperature of the OP-GaAs crystal to $27^{\circ} \mathrm{C}$ to center the gain bandwidth around $10.3 \mu \mathrm{m}$. With both temperature and coarse piezoelectric tuning, we set the idler wavelength at $10,337 \mathrm{~nm}$ with a precision of $\pm 1 \mathrm{~nm}$ with the monochromator. Then we set the OPO wavelength to $10,337.56 \mathrm{~nm}$ using fine piezoelectric actuations. Figure 13 shows the beating of the signal inside the idler pulse when the wavelength emitted by the NesCOPO gets closer to $10,337.56 \mathrm{~nm}$. The top figure shows a beat frequency of $220 \mathrm{MHz}$ measured by Fourier transform. With fine piezo-electric actuation, we managed to tune the idler wavelength as close as $80 \mathrm{MHz}$ from the QCL line at 10,337.56 nm (Fig. 13, bottom).

The instantaneous frequency within the pulse as well as its linewidth can be derived from the beat frequency measurement. To analyse the instantaneous frequency during the pulse, we applied the heterodyne beat-note analysis exposed in Ref. [58] that was already applied on near infrared lasers [59] and OPOs [60]. In a NesCOPO, a frequency chirp within the pulse can be either due to a frequency chirp in the pump pulse or to partial overlap between idler and signal modes as illustrated in Fig. 2. Usually, a large beat frequency of typically a hundred of megahertz is used to generate a large number of oscillations
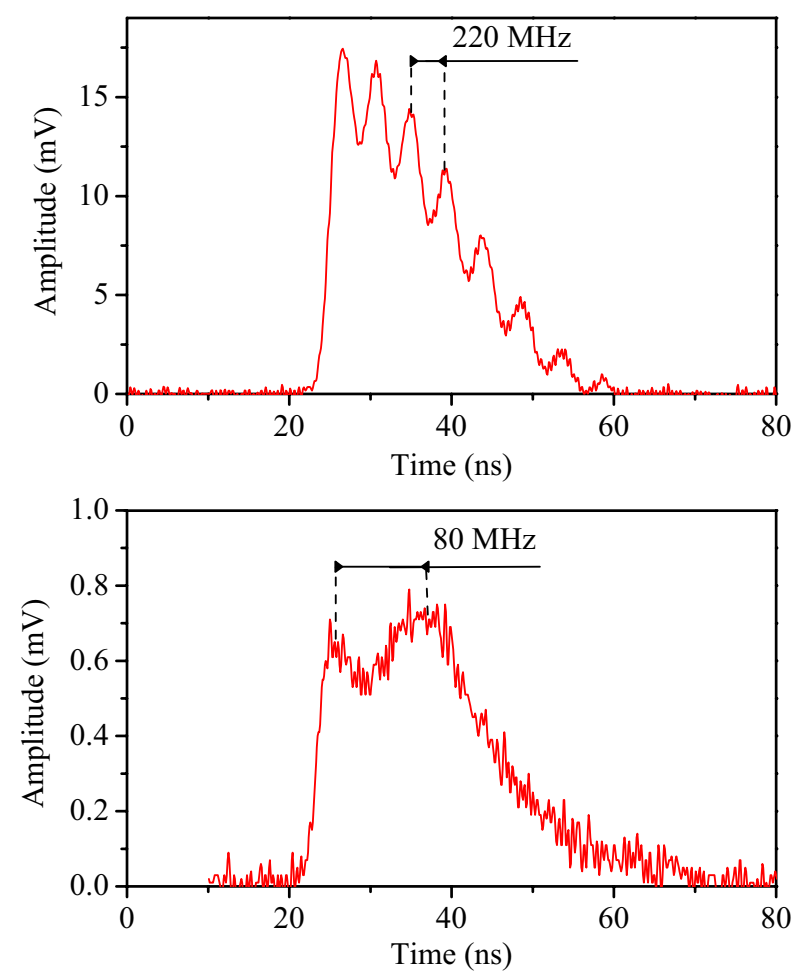

Fig. 13 Frequency beating between the NesCOPO (idler) and a CW DFB-QCL at 10,377.56 nm. Top: $220 \mathrm{MHz}$ and bottom: $80 \mathrm{MHz}$ 
during the optical pulse. It ensures a wide separation of the beat Fourier components and of the coherent pulse. We thus chose the signal obtained in Fig. 13 (top) with a difference frequency of $220 \mathrm{MHz}$, and we adapted the method described in Ref. [59] to retrieve the instantaneous frequency. In fact, due to the asymmetric nature of the idler pulse, it is difficult to extract properly the pulse shape by a simple numerical lowpass filter. Hence, we used the pulse shape obtained without any beat frequency [Fig, 14 (top, red)], and divided the modulated pulse by this shape.

The instantaneous frequency shift can be obtained as explained in Ref. [59]. The oscillating part of the detected signal can be written as follows:

$V(t) \propto 2 E_{\mathrm{QCL}} E_{\mathrm{i}}(t) \cos \left(\omega_{\mathrm{m}} t+\phi(t)\right)$,

where $\omega_{\mathrm{m}}=2 \pi f_{\mathrm{m}}$ is the angular frequency, $E_{\mathrm{QCL}}$ and $E_{\mathrm{i}}$ are the amplitudes of the fields emitted by the QCL and the idler pulse and $\phi(t)$ is the instantaneous phase. Multiplying this relation by either $\cos \left(\omega_{\mathrm{m}} t\right)$ or $\sin \left(\omega_{\mathrm{m}} t\right)$ gives the following system:

$\left\{\begin{array}{l}V(t) \cos \left(\omega_{\mathrm{m}} t\right) \propto E_{\mathrm{i}}(t)\left[\cos \left(2 \omega_{\mathrm{m}} t+\phi(t)\right)+\cos \phi(t)\right] \\ V(t) \sin \left(\omega_{\mathrm{m}} t\right) \propto E_{\mathrm{i}}(t)\left[\sin \left(2 \omega_{\mathrm{m}} t+\phi(t)\right)-\sin \phi(t)\right]\end{array}\right.$
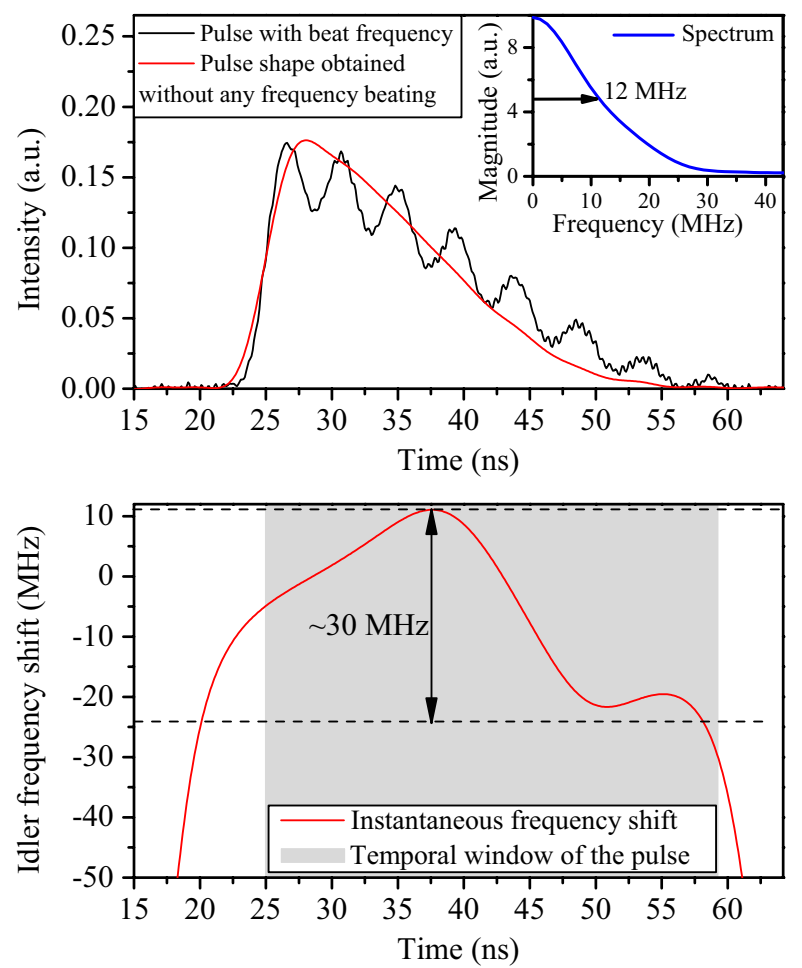

Fig. 14 Top: beat signal between the OPO and the EC-QCL (black) and pulse shaped obtained without beat frequency (red), calculated optical spectrum of the pulse envelope assuming a flat phase for positive frequencies (inset). Bottom: instantaneous frequency shift. Note that the shape of the curve cannot be interpreted as a frequency chirp within the pulse (see the main text for a complete interpretation of this figure)
To get back to the instantaneous phase, we numerically filtered the previous relations by a low-pass filter with a cutoff frequency of $f_{\mathrm{m}} / 4=55 \mathrm{MHz}$. The remaining terms are proportionnal to $E_{\mathrm{i}}(t) \cos (\phi(t))$ and $-E_{\mathrm{i}}(t) \sin (\phi(t))$. Then, we obtained the instantaneous frequency shift defined by:

$\Delta f(t)=\frac{1}{2 \pi} \frac{\mathrm{d} \phi(t)}{\mathrm{dt}}$.

Figure 14 (bottom) shows the calculated instantaneous frequency shift. The measured frequency shift stays within $30 \mathrm{MHz}\left(0.001 \mathrm{~cm}^{-1}\right)$ during the pulse. Besides, the direct Fourier transform of the pulse envelope assuming no chirp has a width of $24 \mathrm{MHz}$. We can thus conclude that there is no significant frequency chirp within the pulse. This makes our source very suitable for detection of CWAs and TICs in the atmosphere which requires linewidths below $0.1 \mathrm{~cm}^{-1}$. Consequently, the limiting factor for general spectroscopy may be the frequency stability rather than the linewidth. Due to the lack of adequate equipment in the LWIR, we were not able to measure the former, but previous experiments with PPLN NesCOPOs have shown that the free running stability is in any case better than $300 \mathrm{MHz}\left(0.01 \mathrm{~cm}^{-1}\right)$ over several minutes [56], while a stability below $10 \mathrm{MHz}$ can be obtained using better opto-mechanics and even below 1 $\mathrm{MHz}$ with servo-locking of the cavity mirrors [51].

\section{Standoff detection of ammonia vapors}

\subsection{Experimental setup}

Figure 15 displays the experimental setup for IP-DIAL measurement of ammonia vapors. The idler is extracted out of the NesCOPO using a $\mathrm{ZnSe}$ dichroic plate at an incidence of $60^{\circ}$ to minimize pump reflection and to maximize idler reflection. A lens of focal length $100 \mathrm{~mm}$ is added to collimate the beam. A long-pass filter with a cut-off wavelength of $6840 \mathrm{~nm}$ is inserted on the path to remove residual pump. The beam passes through a $\mathrm{ZnSe}$ parallel sided plate to divide the idler wave in three paths. The first one, referred to as the measuring path, is obtained by transmission through the plate. Two other reflections on the external and internal faces are used for wavelength analysis and reference path. The transmitted wave is reflected by two goldcoated mirrors towards an afocal system of magnification -2 to reduce beam divergence. The beam passes through a home-made cell with polyethylene windows containing the gas of interest and then hits a sheet of paper placed at $3.5 \mathrm{~m}$. At this distance, the spot is about $10 \mathrm{~mm}$ large. The back scattered light is collected by an off-axis parabolic mirror of focal length $152.4 \mathrm{~mm}$ with an aperture of $68 \mathrm{~mm}$. Note that the collected light does not pass through the cell, so that the measured absorption is single-pass. In addition, a 


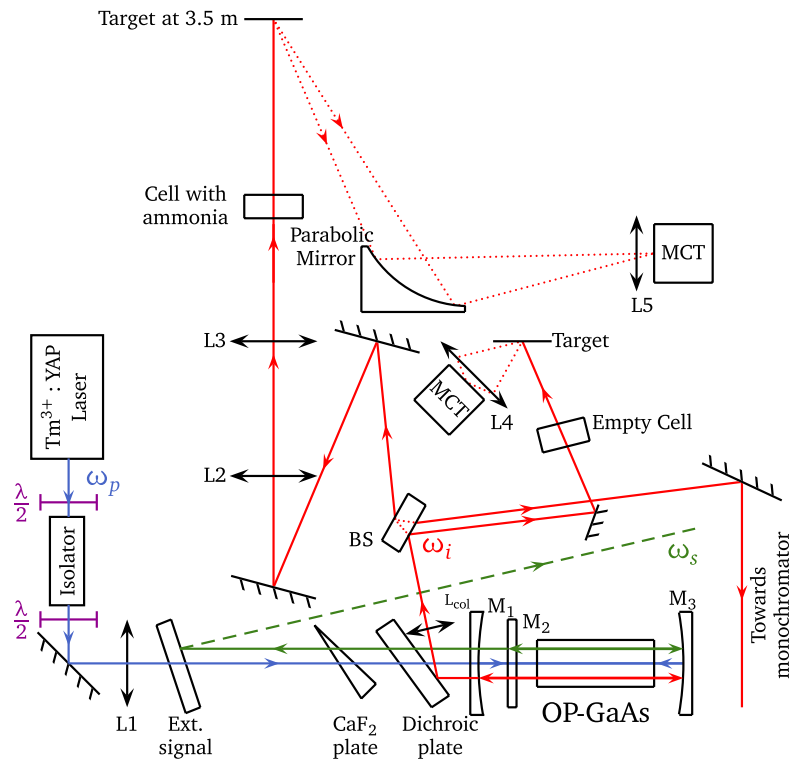

Fig. 15 Experimental IP-DIAL setup for standoff detection

$30 \mathrm{~mm}$ focal length lens was used to increase the field of view, and thus to reduce the magnitude of beam wandering on the detector. A nitrogen-cooled photoconductive $\mathrm{HgCdTe}$ detector with a long-pass filter at $7800 \mathrm{~nm}$ is placed in its focal point. It is followed by a transimpedance amplifier with a measured cut-off frequency of $\sim 300 \mathrm{kHz}$. The reference path is reflected by a mirror towards two paper sheets to create a lambertian reflector. The scattered light is collected by a second $\mathrm{HgCdTe}$ detector with a $20 \mathrm{~mm}$ focal length lens and a long pass filter. Its peak response is at $11.5 \mu \mathrm{m}$, and the measured cut-off frequency is $\sim 100 \mathrm{kHz}$. An empty cell identical to the first one is inserted onto the reference path. Then the reference and the analysis beam experience the same wavelength-dependant losses due to the cell windows, even though polyethylene is supposed to be highly transparent in this spectral region. The third beam is directed with mirrors towards a monochromator (Jobin Yvon) for wavelength analysis. The monochromator resolution is $1 \mathrm{~nm}$ around $10 \mu \mathrm{m}$ i.e. $0.1 \mathrm{~cm}^{-1}$ for the narrowest slits.

\subsection{Reduction of experimental errors}

The single-pass absorption $A$ is calculated as follows:

$A=1-T=1-\frac{S_{\text {analysis }}}{S_{\text {reference }}}$,

where the signals $S_{\text {analysis }}$ and $S_{\text {reference }}$ are taken as the offsetsubstracted amplitudes of the detected peaks, after averaging on the oscilloscope (32 shots average, i.e. $0.32 \mathrm{~s}$ per spectral point). The quantity $S_{\text {analysis }} / S_{\text {reference }}$ is normalized by its mean value at non-absorbed wavelengths. The calculated

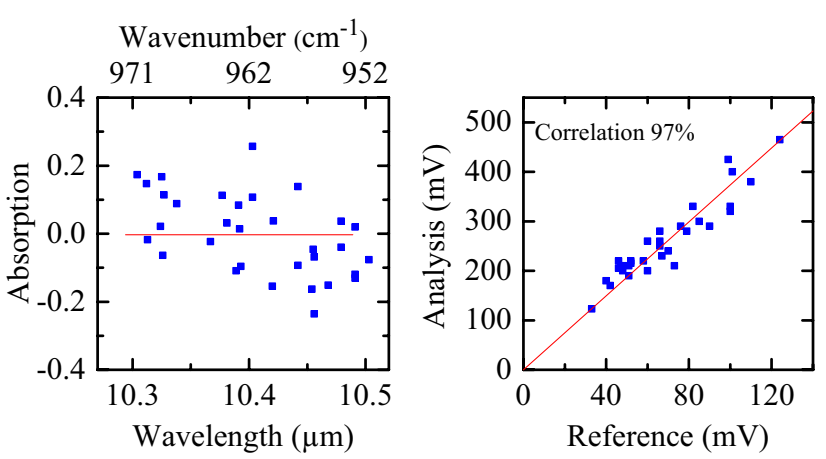

Fig. 16 Left: spectral baseline (without ammonia), with a non-optimized setup. Right: correlation curve of the analysis and reference signal

absorption is independent of the idler intensity, and absorption from atmospheric gases, especially water vapor, is negligible around $10.35 \mu \mathrm{m}$ and for the distances considered here.

The largest source of error in our IP-DIAL measurements lies in fluctuations of the spectrum baseline, i.e. fluctuations independant from ammonia absorption. These fluctuations are specific to standoff sensing using tunable lasers and mainly come from: (1) wavelength-dependent loss, (2) wandering of the beam on the target and/or the detector, and (3) spectral impurities in the output beam. On the one hand, these fluctuations are not random noise, as they cannot be averaged out by integrating over several shots. But on the other hand they are not systematic errors since two baseline spectra acquired in a row seldom give the same result. At the beginning of our experiment we used a non-optimized setup that was slightly different from the one shown in Fig. 15 and measured a sample spectral baseline (without ammonia). These results are shown in Fig. 16. The peak-to-peak fluctuations are $\pm 25 \%$, which is obviously too large for practical measurements. Another way to quantify these fluctuations is to calculate the Pearson's correlation coefficient between the analysis and reference for different signal levels (that are naturally obtained because the output idler energy differs from mode to mode). Even though the relationship between the two signals is linear, as there is no bend in the curve of Fig. 16 (right), the correlation coefficient is only $97 \%$.

To minimize the above-mentioned issues, we have undertaken the following refinements on the non-optimized setup: (1) used similar detectors and filtering optics for the analysis and reference beams, and inserted an empty cell in the reference path, so that spectral dependent losses are identical on both arms, (2) collected the reference flux from scattered light instead of direct beam detection, (3) reduced as much as possible the effective focal length of the collection optics by enlarging the field of view. For instance, the effect of the focal length on the beam wandering is illustrated in Fig. 17. 

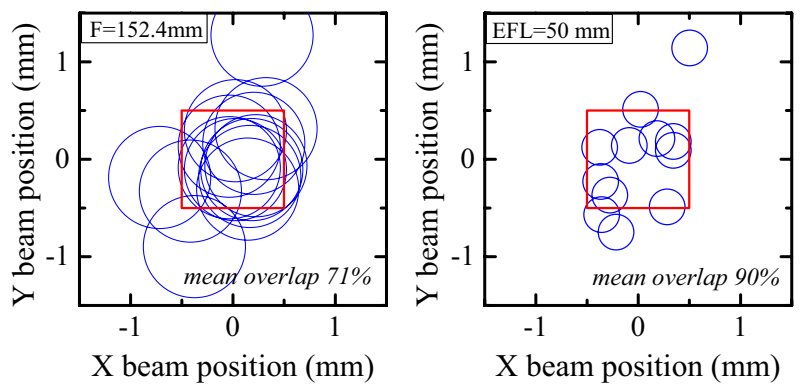

Fig. 17 Beam position on the analysis detector for different idler wavelengths. The spot position is obtained by moving the detector to maximize the signal and noting the displacement on the micrometers. Left: parabolic mirror only. Right: parabolic mirror and field lens. The blue circles have the size of the geometrical image for a $10.5 \mathrm{~mm}$ diameter object at a distance of $3.5 \mathrm{~m}$. The red square represents the MCT detector surface

After each attempt at improving the IP-DIAL setup, we have observed a reduction of the spectral baseline fluctuations. The best result we obtained with the available optics is shown in Fig. 18. The peak-to-peak fluctuations are $\pm 5 \%$ and the correlation coefficient is $99 \%$. In this configuration the effective focal length of the receiving optics is $50 \mathrm{~mm}$ and the reference signal is collected after two diffusions on paper sheets.

We calculated a monochromatic speckle standard deviation of $\pm 3.2 \%$ at $10.3 \mu \mathrm{m}$ using the formula given in Ref. [61] with a $15 \mathrm{~mm}$ beam diameter (1/e) at $3.5 \mathrm{~m}$ and with a $68 \mathrm{~mm}$ receiver aperture. This value corresponds to the lower detection limit for a single longitudinal mode and single transverse mode system. From this, we can conclude that speckle may be a non negligible noise source in our system.

\subsection{Measurement of ammonia spectrum around 10.35 and $10.5 \mu \mathrm{m}$}

Ammonia vapor is obtained with an ammonium hydroxide solution diluted in water at $25 \%$. A few drops are placed in a
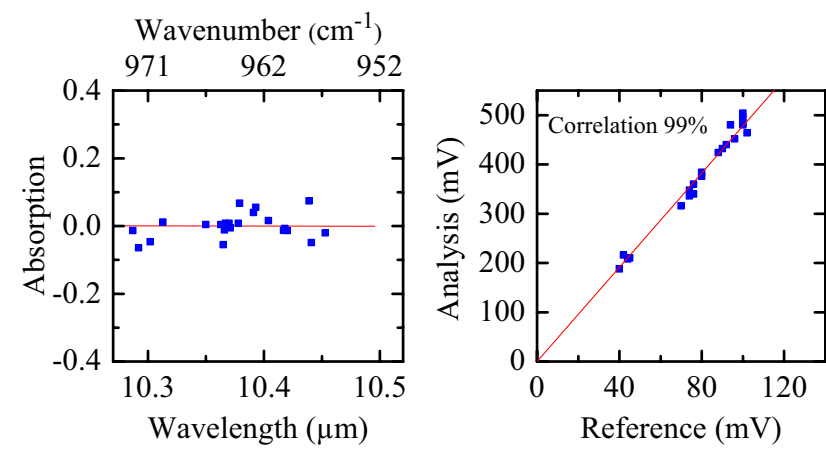

Fig. 18 Left: spectral baseline (without ammonia), with an optimized setup. Right: correlation curve of the analysis and reference signal cell equipped with polyethylene windows. Because ammonia has a high vapor pressure, it evaporates rapidly and fills the cell. The analysis beam passes through the cell and hits the paper target. To acquire the spectrum, the NesCOPO is tuned thanks to the translation of mirrors $\mathrm{M}_{1}$ and $\mathrm{M}_{3}$ via piezoelectric transducers at fixed temperature. The NesCOPO thus sequentially emits the most intense modes under the gain bandwidth, typically five modes. For each mode, the optical absorption is calculated from the ratio of the averaged analysis and reference signals. Once every possible mode has been emitted, the gain curve is shifted by changing the crystal temperature and the fine tuning procedure is repeated. Each wavelength measurement can take a few minutes as we have to manually search for the emitted wavelength with the monochromator, make sure that the emission is stable (meaning that there are no jumps between modes) and that the wavelength is different from the previous ones. Heating and cooling the crystal in order to shift the gain bandwidth can also take a few minutes. An improvement of our source would feature servo-locked mirrors and the use of a PC-driven lambdameter for wavelength measurement to speed up the detection process. However, we would like to stress out that full spectrum acquisition was done to illustrate the tunability and the capability of our source to detect gases. In an operational system, only a few discrete wavelengths would be emitted chosen according to the species, concentration, distance, and possible interfering species which would also speed up the detection process.

Figure 19 shows the absorption line measured between 10.25 and $10.48 \mu \mathrm{m}$. To span this range, the OP-GaAs temperature was increased from 21 to $38^{\circ} \mathrm{C}$. The diffusive target

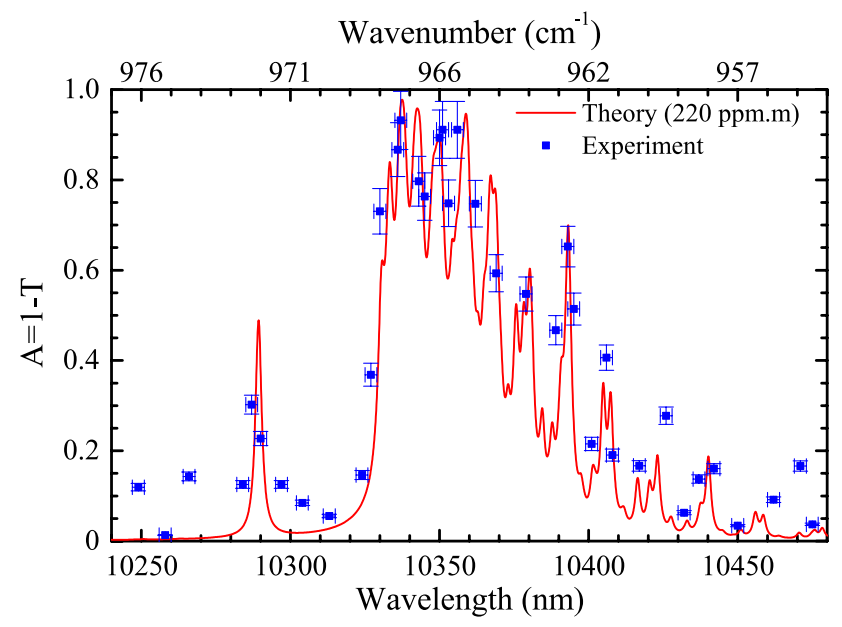

Fig. 19 Measurement of the absorption coefficient of ammonia vapor between 10.25 and $10.48 \mu \mathrm{m}$, in IP-DIAL mode with a target at a distance of $3.5 \mathrm{~m}$. The horizontal error bar corresponds to the monochromator resolution; the vertical error bar integrates the standard error of the analysis and reference channels, as well as the spectral baseline fluctuations 
was placed at a distance of $3.5 \mathrm{~m}$ from the setup. The red line is the absorption spectrum of ammonia for a concentrationlength product of $220 \mathrm{ppm}$ m, calculated using the Hitran database and assuming Voigt profiles for the rotational lines [62]. We supposed the errors to be dominated by the baseline fluctuations, and we derived the vertical error bars using the maximum peak-to-peak value of $\pm 5 \%$ obtained in Fig. 18 (left). The horizontal error bars originate from the resolution of the monochromator, which is $1 \mathrm{~nm}$ around $10 \mu \mathrm{m}$, i.e. $0.1 \mathrm{~cm}^{-1}$.

Finer absorption lines of ammonia can also be resolved, as illustrated in Fig. 20. To obtain this spectrum, the NesCOPO was tuned between 10.492 and $10.528 \mu \mathrm{m}$ through a temperature shift of $2.5^{\circ} \mathrm{C}$.

In these measurements, the wavelength precision is currently limited by the monochromator resolution to about $0.1 \mathrm{~cm}^{-1}$, and by the manual control of the wavelength. Work is under progress to use a commercial lambdameter, which would provide a resolution of $0.01 \mathrm{~cm}^{-1}$ as well as automatic tuning. Regarding the precision on the retrieved concentration, it is actually limited by the fluctuations of the baseline, that we think are mainly due to beam wandering on the detectors in spite of our efforts to optimize the system. This problem could be solved by using an integrating sphere on the reference and by increasing the field of view of the lidar receiver.

To estimate the maximum range of operation of our system, we also performed additional tests. We have measured the amplitude of the backscattered signal as collected by a mirror with an aperture of $254 \mathrm{~mm}$. At a distance of $40 \mathrm{~m}$, the digitized signal voltage was approximately five times lower than the one available during $\mathrm{NH}_{3}$ measurements: yet we are still far from being limited by the detection noise. To increase the range even further, the output energy can be increased by parametric amplification of the idler wave: with

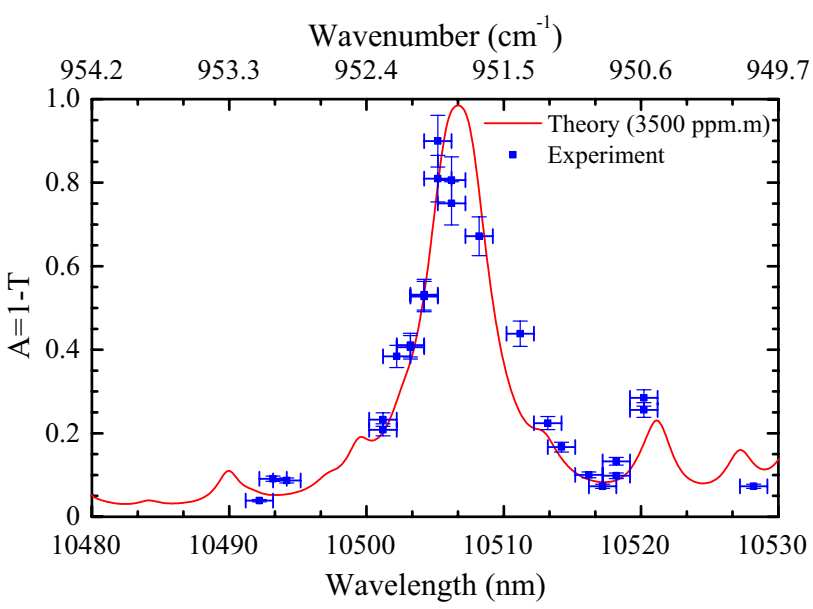

Fig. 20 Measurement of an ammonia vapor absorption line around $10.507 \mu \mathrm{m}$, in IP-DIAL mode with a target at $3.5 \mathrm{~m}$ a 30-mm-long OP-GaAs crystal, pumped by the same laser as the NesCOPO - as only $15 \%$ of the laser output is used to pump the NesCOPO_-we calculated an amplification factor of 30 , corresponding to an energy of $18 \mu \mathrm{J}$. This value is consistent with previously reported parametric amplification gain $[63,64]$. This would increase the detection range by a factor of 5, which gives $200 \mathrm{~m}$. An energy increase would also enable wavelength measurement with a commercial lambdameter, and thus an automatic control of the wavelength as well as a faster tuning, as already demonstrated in similar systems [50].

\section{Conclusion and outlooks}

We have presented a novel source based on a nested-cavity OPO using several orientation-patterned GaAs crystals. Our source emits tunable single-frequency radiation in the 10.2-11.2 and 7.6-8.6 $\mu \mathrm{m}$ ranges with a linewidth below $30 \mathrm{MHz}$ where many chemicals of interest can be targeted. The oscillation threshold can be as low as $2 \mu \mathrm{J}$ with a typical output energy of a few hundreds of nanojoules and an energy efficiency of $15 \%$. Tunability was achieved by thermal control of the crystals and fine adjustment of cavity lengths by piezoelectric stages. We implemented this source in a IPDIAL setup and measured an ammonia vapor absorption band around 10.35 and $10.507 \mu \mathrm{m}$ at a distance of $3.5 \mathrm{~m}$. Other species such as mustard gas could be targeted with the OP-GaAs crystal emitting between 7.6 and $8.6 \mu \mathrm{m}$. We have to emphasize that this source was developed for detection of chemicals, and not as a spectroscopic tool and still needs to be validated in terms of linearity, sensitivity, Allan variance, accuracy, and reproducibility. While we measured a full spectrum of $\mathrm{NH}_{3}$ for illustration purposes, operational detection of chemicals would be done in a different way. Only a restricted number of wavelengths would be chosen according to the expected concentration, distances, and the possible interfering species. Such a feature can be provided thanks to the versatile tunability of our source based on Vernier sampling [50, 65-67].

Increasing the tunability range of the NesCOPO can readily be done by adding more QPM tracks in the crystal. The NesCOPO tunability could also be increased or made faster by tuning the pump wavelength directly, which is made possible thanks to the latest $2 \mu \mathrm{m}$ source developments [68]. In an optical parametric amplifier based on QPM crystals, the tunability can be increased in the same way as in an optical parametric oscillator. An alternative is to design a wideband amplifier using axially chirped QPM gratings, but this comes at the expense of a lower gain, and it has not yet been tested in OP-GaAs [69, 70]. With birefringent crystals, a rather broad and fast tunability can be achieved by rotating the crystal using piezoelectric transducers. However, in low 
birefringent crystals such as $\mathrm{CdSe}$, for example, this rotation leads to a significant beam translation that must be compensated for, or reduced by using several crystals in series with non-overlapping QPM angles. An innovative solution might be to use a hemi-cylindrically polished crystal, which provides wide tunability without misalignment [71].

In a Lidar, the main parameter driving the detection range is the peak power. An external optical amplifier has then to be used to increase the peak power without affecting the spectral and spatial properties of the beam provided by the oscillator. At low energies, a gain of at least 30 can be obtained by OPA in OP-GaAs pumped by a microlaser. To increase the energy even further, a more powerful laser must be used, along with larger aperture crystals such as $\mathrm{ZnGeP}_{2}$ and CdSe. Given the current state of technology, a LWIR energy of $1-10 \mathrm{~mJ}$ with a narrow spectrum should be obtainable in the $8-12 \mu \mathrm{m}$ range. Going beyond $20 \mathrm{~mJ}$ in the LWIR with a narrow spectrum will require crystal apertures larger than $5 \mathrm{~mm}$ with good homogeneity, or coherent combining.

Acknowledgements We acknowledge the French Agence Nationale de la Recherche and Direction Générale de l'Armement for partial funding (ANR-11 ASTR-016). The authors would like to thank Jean-François Lampin and his team from IEMN (Lille, France) for lending us a CW DFB-QCL.

\section{References}

1. R.A. Robinson, P.T. Woods, M.J.T. Milton, Proc. SPIE 2506, 140-149 (1995)

2. P.A. Martin, Chem. Soc. Rev. 31, 201-210 (2002)

3. M.W. Sigrist, R. Bartlome, D. Marinov, J.M. Rey, D.E. Vogler, H. Wächter, Appl. Phys. B Lasers Optics 90, 289-300 (2008)

4. J. Hodgkinson, R.P. Tatam, Meas. Sci. Technol. 24(1), 012004 (2013)

5. G.A. Wagner, D.F. Plusquellic, Appl. Opt. 55(23), 6292-6310 (2016)

6. K.G. Hay, O. Norberg, E. Normand, H. Onnerud, P. Black, Adv. Opt. Technol. 6(2), 67-73 (2017)

7. H. Kariminezhad, P. Parvin, F. Borna, A. Bavali, Opt. Lasers Eng. 48(4), 491-499 (2010)

8. A.S. Grishkanich, A.P. Zhevlakov, V.G. Bespalov, V.V. Elizarov, S.V. Kascheev, A.A. Ilinskiy, Proc. SPIE 9274, 92741L (2014)

9. M.E. Webber, M. Pushkarsky, C.K.N. Patel, J. Appl. Phys. 97, 11 (2005)

10. C.W. Van Neste, L.R. Senesac, T. Thundat, Anal. Chem. 81(5), 1952-1956 (2009)

11. B.J. Orr, Infrared LIDAR Applications in Atmospheric Monitoring (Wiley, Oxfrod, 2006)

12. J. Kadlčák, Proceedings of the 5th International Symposium Protection Against Chemical and Biological Warfare Agents p. 237 (1995)

13. P. Adam, J.L. Duvent, S.W. Gotoff, Proc. SPIE 3127, 212-223 (1997)

14. J.V. Cernius, D.A. Elser, J. Fox, Proc. SPIE 1062, 164-171 (1989)

15. D.B. Cohn, E.J. Griffin, L.F. Klaras, M.E. Ehritz, C.R. Swim, J.A. Fox, Proc. SPIE 4378, 34-42 (2001)

16. C .B. Carlisle, J .E. van derLaan, L.W. Carr, P. Adam, J .P. Chiaroni, Appl. Opt. 34(27), 6187-6200 (1995)
17. Stand-Off Detector DD-CWA-SM. http://www.gammatech.hu

18. A. Pal, C.D. Clark, M. Sigman, D.K. Killinger, Appl. Opt. 48(4), B145-B150 (2009)

19. A. Mukherjee, S.V. der Porten, C.K.N. Patel, Appl. Opt. 49(11), 2072-2078 (2010)

20. R.F. Curl, F. Capasso, C. Gmachl, A.A. Kosterev, B. McManus, R. Lewicki, M. Pusharsky, G. Wysocki, F.K. Tittel, Chem. Phys. Lett. 487(1-3), 1-18 (2010)

21. C.W. Van Neste, L.R. Senesac, T. Thundat, Appl. Phys. Lett. 92(23), 234102 (2008)

22. F. Fuchs, S. Hugger, M. Kinzer, R. Aidam, W. Bronner, R. Lösch, Q. Yang, K. Degreif, F. Schnürer, Opt. Eng. 49(11), 111127-111127 (2010)

23. X. Chen, D. Guo, F.S. Choa, C.C. Wang, S. Trivedi, A.P. Snyder, G. Ru, J. Fan, Appl. Opt. 52(12), 2626-2632 (2013)

24. A. Goyal, T. Myers, C.A. Wang, M. Kelly, B. Tyrrell, B. Gokden, A. Sanchez, G. Turner, F. Capasso, Opt. Express 22(12), 14392-14401 (2014)

25. Q. Hu, J.S.K. Lim, H. Liu, Y. Fu, Opt. Express 24(17), 1914819156 (2016)

26. N.A. Macleod, F. Molero, D. Weidmann, Opt. Express 23(2), 912-928 (2015)

27. J. Sun, J. Ding, N. Liu, G. Yang, J. Li, Spectrochim. Acta Part A Mol. Biomol. Spectrosc. 191, 532-538 (2018)

28. J.S. Li, B. Yu, H. Fischer, W. Chen, A.P. Yalin, Rev. Sci. Instrum. 86(3), 031501 (2015)

29. A. Bakkland, H. Fonnum, E. Lippert, M.W. Haakestad, in: Conference on Lasers and Electro-Optics (OSA 2016), pp. 25-26

30. S. Chandra, T.H. Allik, G. Catella, R. Utano, J.A. Hutchinson, Appl. Phys. Lett. 71(5), 584-586 (1997)

31. R.K. Feaver, R.D. Peterson, P.E. Powers, Opt. Express 21(13), 16104-16110 (2013)

32. A. Tyazhev, D. Kolker, G. Marchev, V. Badikov, D. Badikov, G. Shevyrdyaeva, V. Panyutin, V. Petrov, Opt. Lett. 37(19), 4146-4148 (2012)

33. S. Vasilyev, S. Schiller, A. Nevsky, A. Grisard, D. Faye, E. Lallier, Z. Zhang, a J Boyland, J .K. Sahu, M. Ibsen, W .A. Clarkson, Opt. Lett. 33(13), 1413-1415 (2008)

34. J. Armougom, J.M. Melkonian, J.B. Dherbercourt, M. Raybaut, A. Godard, R.S. Coetzee, A. Zukauskas, V. Pasiskevicius, 2017 European Conference on Lasers and Electro-Optics and European Quantum Electronics Conference p. CD P.32 (2017)

35. V. Vaicikauskas, V. Kabelka, Z. Kuprionis, M. Kaucikas, Proc. SPIE 5988, 5988-5988-6 (2005)

36. G. Mennerat, P. Kupecek, Advanced Solid State Lasers (OSA, 1998), pp. $15-18$

37. J. Yuan, Y. Chen, X. Duan, B. Yao, T. Dai, Y. Ju, Opt. Laser Technol. 92, 1-4 (2017)

38. T. Skauli, K.L. Vodopyanov, T.J. Pinguet, A. Schober, O. Levi, L.A. Eyres, M.M. Fejer, J.S. Harris, B. Gerard, L. Becouarn, E. Lallier, G. Arisholm, Opt. Lett. 27(8), 628-630 (2002)

39. E. Lallier, M. Brevignon, J. Lehoux, Opt. Lett. 23(19), 15111513 (1998)

40. A. Grisard, E. Lallier, B. Gérard, Opt. Mater. Express 2(8), 1020-1025 (2012)

41. C. Kieleck, M. Eichhorn, A. Hirth, D. Faye, E. Lallier, Opt. Lett. 34(3), 262-264 (2009)

42. P.S. Kuo, K.L. Vodopyanov, M.M. Fejer, D.M. Simanovskii, X. Yu, J.S. Harris, D. Bliss, D. Weyburne, Opt. Lett. 31(1), 71-73 (2006)

43. K.L. Vodopyanov, O. Levi, P.S. Kuo, T.J. Pinguet, J.S. Harris, M.M. Fejer, B. Gerard, L. Becouarn, E. Lallier, Opt. Lett. 29(16), 1912-1914 (2004)

44. K.L. Vodopyanov, I. Makasyuk, P.G. Schunemann, Opt. Express 22(4), 4131-4136 (2014) 
45. J. Wueppen, S. Nyga, B. Jungbluth, D. Hoffmann, Opt. Lett. 41(18), 4225-4228 (2016)

46. Q. Clément, J.M. Melkonian, J.B. Dherbecourt, M. Raybaut, A. Grisard, E. Lallier, B. Gérard, B. Faure, G. Souhaité, A. Godard, Opt. Lett. 40(12), 2676-2679 (2015)

47. A. Godard, M. Raybaut, M. Lefebvre, Nested Cavity Optical Parametric Oscillators-A Tunable Frequency Synthesizer for Gas Sensing, Chap. Environment: Trace Gas Monitoring (Wiley, Oxford, 2006)

48. A. Berrou, M. Raybaut, A. Godard, M. Lefebvre, Appl. Phys. B 98 (2010)

49. J. Bjorkholm, A. Ashkin, R. Smith, IEEE J. Quantum Electron. 6(12), 797-799 (1970)

50. J. Barrientos Barria, A. Dobroc, H. Coudert-Alteirac, M. Raybaut, N. Cézard, J.B. Dherbecourt, T. Schmid, B. Faure, G. Souhaité, J. Pelon, J.M. Melkonian, A. Godard, M. Lefebvre, Appl. Phys. B 117(1), 509-518 (2014)

51. J. Barrientos-Barria, D. Mammez, E. Cadiou, J.B. Dherbecourt, M. Raybaut, T. Schmid, A. Bresson, J.M. Melkonian, A. Godard, J. Pelon, M. Lefebvre, Opt. Lett. 39(23), 6719-6722 (2014)

52. J.A. Giordmaine, R.C. Miller, Phys. Rev. Lett. 14(24), 973-976 (1965)

53. B. Scherrer, I. Ribet, A. Godard, E. Rosencher, M. Lefebvre, J. Opt. Soc. Am. B 17(10), 1716 (2000)

54. M.M. Fejer, G.A. Magel, D.H. Jundt, R.L. Byer, IEEE J. Quantum Electron. 28(11), 2631-2654 (1992)

55. A. Grisard, B. Faure, G. Souhaité, E. Lallier, Adv. Solid State Lasers, ATu2A.39 (2014)

56. B. Hardy, A. Berrou, S. Guilbaud, M. Raybaut, A. Godard, M. Lefebvre, Opt. Lett. 36(5), 678-680 (2011)

57. S.L. Bartelt-Hunt, D.R.U. Knappe, M.A. Barlaz, Crit. Rev. Environ. Sci. Technol. 38(2), 112-136 (2008)
58. R.T. White, Y. He, B.J. Orr, M. Kono, K.G.H. Baldwin, J. Opt. Soc. Am. B 21(9), 1577-1585 (2004)

59. L. Cabaret, C. Drag, Eur. Phys. J. Appl. Phys. 51(2), 20702 (2010)

60. M. Wirth, A. Fix, P. Mahnke, H. Schwarzer, F. Schrandt, G. Ehret, Appl. Phys. B 96(1), 201 (2009)

61. J.A. Fox, C.R. Gautier, J.L. Ahl, Appl. Opt. 27(5), 847-855 (1988)

62. Y. Liu, J. Lin, G. Huang, Y. Guo, C. Duan, J. Opt. Soc. Am. B 18(5), 666-672 (2001)

63. G. Bloom, A. Grisard, E. Lallier, C. Larat, M. Carras, X. Marcadet, Opt. Lett. 35(4), 505-507 (2010)

64. Q. Clément, J.M. Melkonian, J. Barrientos-Barria, J.B. Dherbecourt, M. Raybaut, A. Godard, Opt. Lett. 38(20), 4046-4049 (2013)

65. B. Hardy, M. Raybaut, J.B. Dherbecourt, J.M. Melkonian, A. Godard, A.K. Mohamed, M. Lefebvre, Appl. Phys. B 107(3), 643-647 (2012)

66. E. Cadiou, D. Mammez, J.B. Dherbecourt, G. Gorju, J. Pelon, J.M. Melkonian, A. Godard, M. Raybaut, Opt. Lett. 42(20), 4044-4047 (2017)

67. J. Armougom, J.M. Melkonian, M. Raybaut, J.B. Dherbecourt, G. Gorju, A. Godard, R. Cotzee, V. Pašiškevičius, J. Kadlčák, High-Brightness Sources and Light-driven Interactions, p. MM4C.7 (2018)

68. F. Gutty, A. Grisard, A. Joly, C. Larat, D. Papillon-Ruggeri, E. Lallier, Opt. Express 23(5), 6754-6762 (2015)

69. M. Charbonneau-Lefort, B. Afeyan, M.M. Fejer, J. Opt. Soc. Am. B 25(4), 463-480 (2008)

70. Z.G. Figen, O. Aytür, O. Arikan, Appl. Opt. 55(9), 2404-2412 (2016)

71. V. Kemlin, D. Jegouso, J. Debray, E. Boursier, P. Segonds, B. Boulanger, H. Ishizuki, T. Taira, G. Mennerat, J.M. Melkonian, A. Godard, Opt. Express 21(23), 28886-28891 (2013) 